Michael Ringhofer

Christoph J. Brabec

Rudolf Sobczak

Dave Mead

John Driscoll

\section{Investigation of the linear flow regime of commercial polymers by numerical conversion of MVM creep measurements}

Received: 30 January 1997

Accepted: 8 September 1997
M. Ringhofer - C. J. Brabec

R. Sobczak (

Institute of Chemistry/Physical Chemistry

Johannes Kepler University Linz

Altenbergerstr. 69

A-4040 Linz, Austria

D. Mead $\cdot$ J. Driscoll

Department of Mechanical Engineering

University of Michigan

Ann Arbor

Michigan 48109, USA
Abstract A new experimental and numerical method has been developed to characterize the terminal flow behavior of polydisperse, commercial grade polymer melts over a wide dynamic range of time/frequency scales. Experimentally, an MVM rheometer specifically designed for long time scale $\left(t \sim 10^{4} \mathrm{~s}\right)$ creep measurements is used to measure the creep compliance of three commercial polymers: two high density polyethylenes and one polystyrene. The long time scale MVM creep data are complemented in the short time scale regime by creep data from an industrial plate-plate rheometer. The timedependent creep data is combined and converted to a discrete retardation spectra using a nonlinear regularization algorithm to address the ill-posed nature of the interconversion. The retardation spectrum is analytically converted to dynamic moduli and compared with independently measured dynamic moduli. In the overlapping frequency region, calculations and measurements show excellent agreement and the combined data span a much larger dynamic range than either independent data set. The calculated and measured dynamic moduli data are combined and a retardation spectrum with a vastly expanded dynamic range is generated. Combining long time scale MVM creep compliance data and dynamic moduli data exploits the intrinsic sensitivities of controlled strain and controlled stress rheological experiments and is a powerful means to greatly expand the experimentally accessible dynamic range of time/frequency. This approach is particularly useful for commercial polymers with broad molecular weight distributions and commensurately large distributions of relaxation times.

Key words Retardation time spectra - creep measurements compliance - MVM - regularization

\section{Introduction}

The rapid development of metallocene-catalyzed polymers has made it possible to carefully control the molecular weight distribution of prosaic commercial polymers such as polyethylene and polypropylene (Thayer, 1995). For linear flexible polymers, the molar mass distribution is the most fundamental characteristic of the material. In principle, knowledge of the molar mass dis- tribution is equivalent to knowledge of all mechanical properties of the melt (Doi and Edwards, 1986). Thus, the ability to manipulate the molecular weight distribution imparts the ability to manipulate the physical properties of the melt and hence its processing characteristics. The enhanced chemical capabilities of metallocene catalysts has brought about a need for improved material characterization techniques with enhanced sensitivity and resolution of subtle changes in the molecular 
weight distribution. Relatively simple characterization methods currently in use such as melt flow index (MFI) do not have the ability to resolve relatively small differences in molecular weight distribution that can be readily produced with metallocenes.

The extreme sensitivity of linear viscoelastic material functions with respect to molecular weight and molecular weight distribution coupled with the simplicity of the measurements make them ideal properties for characterizing polymers with complex molecular weight distributions. For example, the zero shear viscosity is known to scale as weight average molecular weight to the 3.4 power. Thus, small changes in the molecular weight distribution lead to large changes in viscosity. Other linear viscoelastic material properties and functions have similarly strong dependence on molecular weight and/or its dispersion. Indeed, the extreme sensitivity to molecular weight is the fundamental principle that underlies rheological methods of determining the molecular weight distribution (Tsenoglou, 1991; Mead, 1994).

There are inherent experimental problems associated with measuring the relaxation spectrum of commercial polymers that are principally associated with the large dynamic range of time/frequency required to characterize these systems. A rough estimate of the dynamic range required for a complete characterization of the linear viscoelastic relaxation spectrum can be gleaned via a simple calculation based on the scaling of the longest terminal relaxation time with molecular weight. The longest terminal relaxation time scales as molecular weight to the 3.4 power, $\lambda \sim \mathrm{M}^{3.4}$. If a commercial polymer has a molecular weight distribution spanning $\sim 3$ decades, which is not uncommon, the corresponding relaxation spectrum will span at least $\sim 10$ decades of time/frequency! This is truly an enormous dynamic range and no single rheological instrument possesses such a huge dynamic range. Frequently, time/temperature superposition is used to expand the effective dynamic range of rheological instruments. However, for many common polymer systems, such as commercial polyolefins like PE or PP, the activation energy for flow is so small that time/temperature superposition cannot be effectively used to significantly enlarge the dynamic range of measurement (Janeschitz-Kriegl, 1983).

Physically, stress in the terminal zone is due principally to the high molecular weight tail of the molecular weight distribution. Although the high molecular weight tail may contribute a relatively small fraction of the total stress throughout most of the linear viscoelastic frequency domain, their contribution in fast non-linear flows, where processing occurs, is usually very significant. In fact, the contribution of the high molecular weight tail can completely alter the qualitative behavior of the flow. Specifically, the long time scale relaxation processes associated with the high molecular weight tail control the elastic character of the melt and are intimately related to important processing phenomena such as die swell, bubble stability in film blowing and elastically derived flow instabilities. Thus, to properly characterize the processability of the melt, it is crucially important to access the full terminal zone behavior where the high molecular weight tail of the molecular weight distribution dominates.

Beyond the issue of dynamic range limitations, there are also serious considerations about the ability to experimentally resolve the minute stress levels present in the terminal zone of polydisperse systems. Limitations in transducer technology will always set an upper bound on the accessible relaxation times in any controlled strain experiment. Since no single rheological instrument is capable of measuring such a wide dynamic range of both time/frequency and stress (torque), it will generally be necessary to make multiple measurements with different instruments and combine the data if a complete spectrum spanning the entire terminal and plateau regions is to be obtained. In this regard, it has long been known that certain linear viscoelastic experiments are more/less sensitive to the long/short timescales than others. Specifically, it is generally known that constrained elastic recovery experiments are much more sensitive to long time scale relaxation processes than dynamic moduli measurements (Plazek, 1992; Plazek and Chay, 1991). Qualitatively, this can be understood since, in the zero Reynolds number limit, even the smallest residual stress is, in principle, capable of driving the strain recovery process. Ultimately, the experiment will be limited by parasitic torque and rotational inertia, however, this technique is nevertheless capable of accessing stress levels vastly lower than those in a controlled strain experiment (Franck, 1985). The principal point here is that to access long time scale relaxation in the terminal zone one must utilize an experimental method capable of probing the dynamics of a material with truly minute stress levels. One instrument capable of such sensitive measurements is the MVM rheometer which we shall utilize in our work in combination with more conventional rheometers.

One of the principal objectives of this work is to develop viable and rigorous numerical methods to combine and utilize the data from different linear viscoelastic experiments to generate a composite relaxation spectrum with an expanded dynamic range, particularly in the low frequency terminal zone. As noted above, a suitable pair of material functions would be the dynamic moduli $G^{*}(\omega)$ and the creep compliance $J(t)$ which have very different sensitivities to short and long time scale relaxation processes. The issues here are subtle in that measurements from two or more different instruments must be combined such that the data from different experiments interleave smoothly in the overlapping time/frequency regions. Although methods to 
interconvert complete material functions have been developed (Mead, 1994), the specific issues required to interconvert and combine partial data sets have not been previously addressed in the research literature. One possibility to generate viscoelastic mastercurves has been outlined by Kaschta and Schwarzl (1994). For a narrow molar mass distribution polystyrene, they successfully converted creep compliance measurements to dynamic moduli. The agreement between the measurement and numerical conversion was excellent.

In a previous paper, we presented a new experimental method to measure the $\eta^{+}(t)$-material function in long time regime (Ringhofer et al., 1996). A special type of falling sphere viscometer, the magneto viscometer, was adapted to measure time-dependent compliance for extremely long time scales $\left(t \sim 10^{4} \mathrm{~s}\right)$. This instrument is capable of measuring viscosity as well as compliance. The high precision of the instrument is absolutely essential in the terminal flow regime where the stress levels are very low. Comparison with creep compliance measurements from a standard plate-plate theometer (which we label as DSR compliance or DSR creep data) verifies the MVM results. The DSR data are used to complement the MVM creep data in the shorter time scale regions.

In this work we will present MVM creep compliance measurements for three different polydisperse commercial polymers, two HDPE and one polystyrene. The unique ability of the MVM to characterize the long time scale relaxation regime of polymers with high accuracy can be exploited to create creep mastercurves that supplement the DSR creep data. We will demonstrate that MVM measurements provide valuable information about long time scale relaxation processes of commercial polymers, which is otherwise very difficult to access. These data can be combined with experimental dynamic moduli to further expand the experimentally accessible dynamic range of frequency/time by several decades.

This paper is organized as follows: In the second section the strategies and numerical tools for the interconversion of viscoelastic material functions are detailed. The next two sections contain details of the materials and experimental methods employed for the measurement of the time-dependent creep compliance and the dynamic moduli. In the fifth section, results for the creep compliance from different measurement techniques are shown. The interconversion of creep data to dynamic moduli is performed and dynamic mastercurves for all the materials are generated by overlaying the interconverted and measured moduli. Results of the conversions will also be discussed. In the final section we will summarize the unique role of MVM controlled stress creep measurements for the characterization of the terminal behavior of broad molecular weight distribution commercial polymers.

\section{Retardation spectra and their numerical interconversion to dynamic moduli}

Nonlinear regularization for the calculation of retardation spectra

The retardation time spectra - continuous or discrete is a very powerful tool in the characterization of polymer melts (Plazek, 1992). Knowledge of the retardation spectra in principle enables one to calculate any other linear viscoelastic material function (Kaschta and Schwarzl, 1994; Mead, 1994). Furthermore, the spectra contain detailed information about the polymer's molecular weight distribution (Mead, 1994). The retardation spectra are particularly sensitive to contributions from the high molecular weight tail of the distribution.

The retardation time spectrum is generally only accessible to experimental measurement in a very limited range of time scales. Therefore, to determine a spectrum over a broad dynamic range, one has to resort to indirect measurements involving other material functions. One material function which can be measured over a broad range of times with high accuracy is the creep compliance $J(t)$. To extract the retardation time spectrum from experimental data we have to restrict ourselves to the range of small stress such that the linear Boltzmann constitutive equation is valid:

$$
\gamma(t)=\int_{-\infty}^{t} J\left(t-t^{\prime}\right) \dot{\sigma}\left(t^{\prime}\right) d t^{\prime}
$$

Here, $\gamma(t)$ and $\dot{\sigma}(t)$ are the strain and the stress derivative with respect to time, respectively. $J\left(t-t^{\prime}\right)$ is the time-dependent compliance. For creep experiments performed with a Magnet Viscometer (MVM) $(\sigma=0$ for $t<0$ and $\sigma=\sigma_{0}$ for $t>0$ ), Eq. (1) yields

$$
\gamma(t)=J(t) \sigma_{0} \quad t \geq 0
$$

To determine a continuous retardation spectrum $j(\tau)$ for a general stress history Eq. (1) can be transformed into an expression explicit in the compliance (Tschoegl, 1989).

$$
J(t)=J_{0}+\frac{t}{\eta_{0}}+\int_{-\infty}^{+\infty} L(\tau)\left(1-e^{-\frac{t}{\tau}}\right) d \ln (\tau)
$$

Here $L(\tau)$ denotes the nonnegative logarithmic retardation spectrum, e.g. $L(\tau)=\tau j(\tau) . J_{0}$ is the elastic compliance and $\eta_{0}$ the zero shear viscosity. Taking the Fourier transform of (3) leads to the following expressions for the dynamic compliance,

$$
J^{\prime}(\omega)=J_{0}+\int_{-\infty}^{+\infty} L(\tau)\left(\frac{1}{1+\omega^{2} \tau^{2}}\right) d \ln (\tau)
$$




$$
J^{\prime \prime}(\omega)=\frac{1}{\omega \eta_{0}}+\int_{-\infty}^{+\infty} L(\tau)\left(\frac{\omega \tau}{1+\omega^{2} \tau^{2}}\right) d \ln (\tau)
$$

A generalized $N$ mode serial Voigt model (Ferry, 1980; Janeschitz-Kriegl, 1983; Tschoeg1; 1989) is commonly used to approximate the retardation spectrum in (4) with a discrete series of $N$ retardation strengths and retardation times $\left\{J_{k}, \tau_{k}\right\}$. Using the discrete spectrum in (3) and (4) yields

$$
\begin{aligned}
& J(t)=J_{0}+\frac{t}{\eta_{0}}+\sum_{k=1}^{N} J_{k}\left(1-e^{-\frac{t}{\tau_{k}}}\right) \\
& J^{\prime}(\omega)=J_{0}+\sum_{k=1}^{N} J_{k}\left(\frac{1}{1+\omega^{2} \tau_{k}^{2}}\right) \\
& J^{\prime \prime}(\omega)=\frac{1}{\omega \eta_{0}}+\sum_{k=1}^{N} J_{k}\left(\frac{\omega \tau}{1+\omega^{2} \tau_{k}^{2}}\right)
\end{aligned}
$$

A similar set of equations describes the relationship between the time-dependent or dynamic moduli $G(t)$, $G^{\prime}(\omega), G^{\prime \prime}(\omega)$ and the relaxation time spectrum.

From Eqs. (5) one can see that the numerical calculation of a discrete retardation spectrum requires the determination of a total of $2 N+2$ parameters. The solution of these equations is an ill-posed problem according to a definition of Hadamard (Morozov, 1984). An operational characteristic of the ill-posedness is that small uncertainties in the experimental data may lead to large fluctuations and errors of the retardation time spectrum (Friedrich and Hoffmann, 1983; Honerkamp and Weese, 1989). Therefore, substantial numerical effort is typically necessary to determine the response spectrum from time-dependent creep data.

During the past few years considerable progress has been made in the development of numerical routines to solve ill-posed problems in rheology. All methods to address the ill posed nature of systems pivot on introducing additional a priori information on the structure of the solution into the algorithm. There are two major classes of numerical methods which have been successful in the calculation of relaxation time spectra. In the first category we include algorithms based on standard linear or non-linear regression analysis. Representatives of this class are the algorithms from Kaschta (1992), Emri and Tschoegl (1993, 1994), Baumgaertel and Winter (1989) and Brabec and Schausberger (1995). The second category is regularization analysis which gains more significance as the computational power of PCs increases. There exist different implementations of regularization algorithms like CONTIN (Provencher, 1982; Mead, 1994) or NLREG (Honerkamp and Weese, 1993; Weese, 1992) and FTIKREG (Honerkamp and Weese,
1990; Honerkamp et al., 1991), but the basic principles of the numerical algorithms are all identical. Since this paper deals with the numerical calculation of retardation time spectra and their interconversion to linear material functions, analytical approximations (Schwarzl and Staverman, 1952; Ferry, 1980) will not be considered.

Regularization programs, like that of Honerkamp and Weese, do not have to restrict the number of relaxation times to guarantee the calculation of reasonable shaped, smooth spectra. Tikhonov regularization eliminates high frequency oscillations by penalizing the residual of the error norm with functions proportional to the norm of the spectrum or its second derivative. An additional parameter $\alpha$, the regularization parameter, is introduced to control the magnitude of the curvature penalty function in Eq. (6),

$$
\begin{aligned}
V(a)= & \sum_{i=1}^{N} \frac{1}{\sigma_{i}^{2}} \\
& \cdot\left[J_{i}^{\sigma}-\left(J_{0}+\frac{t}{\eta_{0}}+\int_{-\infty}^{+\infty}\left(1-e^{-\frac{1}{\tau}}\right) L(\tau) d(\ln \tau)\right)\right]^{2} \\
& +a \int_{-\infty}^{+\infty}\left(\frac{d^{2}}{d \tau^{2}} L(\tau)\right)^{2} d(\ln \tau)
\end{aligned}
$$

where $J_{1}^{\sigma}, \ldots, J_{N}^{\sigma}$ denote the experimental data for $J\left(t_{1}\right), \ldots, J\left(t_{N}\right)$ and $\sigma_{1}, \ldots, \sigma_{N}$ are the corresponding errors.

Further improvements in the quality of spectra calculations were realized with the development of nonlinear regularization, which calculates the logarithm of the spectrum $\tilde{L}(\tau)=\log L(\tau)$ instead of the spectrum $L(\tau)$ itself. For the calculation of the logarithm of a spectrum from creep data, Eq. (3) is modified in the following way leading to a new representation (7)

$$
J(t)=J_{0}+\frac{t}{\eta_{0}}+\int_{-\infty}^{+\infty} 10^{\tilde{L}(\tau)}\left(1-e^{-\frac{t}{\tau}}\right) d \ln (\tau)
$$

and a new objective functional (8)

$$
\begin{aligned}
V(a)= & \sum_{i=1}^{N} \frac{1}{\sigma_{i}^{2}} \\
& \cdot\left[J_{i}^{\sigma}-\left(J_{0}+\frac{t}{\eta_{0}}+\int_{-\infty}^{+\infty}\left(1-e^{-\frac{t}{\tau}}\right) 10^{\tilde{L}(\tau)} d(\ln \tau)\right)\right]^{2} \\
& +a \int_{-\infty}^{+\infty}\left(\frac{d^{2}}{d \tau^{2}} \tilde{L}(\tau)\right)^{2} d(\ln \tau)
\end{aligned}
$$


The regularization parameter is determined in a selfconsistent manner. A detailed description of the algorithm and its implementation can be found in the original literature of Weese (1992). To obtain a discrete retardation spectrum $J_{k}$ and $\tau_{k}$, a set of parameters $\tau_{k}$ has to be prescribed. The retardation times $\tau_{k}$ in our case were pre-specified to be logarithmically equidistant with spacings of up to 10 retardation times per decade. Therefore, minimization of Eq. (8) determines $N+2$ parameters, $N$ retardation weights $J_{k}$ as well as $J_{0}$ and $\eta_{0}$. For all materials regularization calculated positive, smooth, physical reasonable spectra with no residual dependencies on the number or the density of retardation times. The average deviation between the experimental and recalculated data was smaller than the experimental error in the creep measurements, indicating that no further improvement to the fit was possible.

The preservation of the higher integral moments of the spectrum is a powerful criteria to assess the physical relevance of solutions for ill-posed problems. This is especially important in the case of retardation or relaxation time spectra, where the higher integral moments are quantities, such as the zero shear viscosity and recoverable compliance, which can be directly and independently accessed by experiment (Mead, 1994). For the retardation spectrum, self-consistency demands that the following relation be satisfied:

$$
J_{e}^{0}=\frac{1}{G_{N}^{0}}+\sum_{k=1}^{N} J_{k}
$$

This point is particularly important since the retardation spectrum will be used for numerical interconversion between creep compliance and dynamic moduli. For successful interconversions, the retardation spectrum has to contain the correct characteristic information of the polymer system. It has recently been demonstrated (Brabec et al., 1997) that the nonlinear regularization algorithm is capable of preserving a spectrum's higher integral moments. Therefore nonlinear regularization will calculate retardation spectra containing equivalent selfconsistent information such as other linear viscoelastic material functions.

Determination of the dynamic moduli from creep compliance

The relaxation modulus $G(t)$ and the creep compliance $J(t)$ are related to one another by the Volterra equation (Ferry, 1980)

$$
t=\int_{-\infty}^{+\infty} J\left(t^{\prime}\right) G\left(t-t^{\prime}\right) d t^{\prime}
$$

If one of the functions $G(t)$ or $J(t)$ is known, the other function can in principle be calculated by inverting the convolution Eq. (10). Since this process requires inverting LaPlace transforms, the interconversion between $G(t)$ and $J(t)$ via this route is also an ill-posed problem. However, when either $G(t)$ or $J(t)$ is given in discrete form, the interconversion between them is algebraic and trivial (Mead, 1994). In the frequency domain, the relationship between $G^{*}(\omega)$ and $J^{*}(\omega)$ are algebraic and the conversion between the dynamic moduli and compliance is trivial;

$$
\begin{aligned}
& G^{\prime}(\omega)=\frac{J^{\prime}(\omega)}{\left[J^{\prime}(\omega)\right]^{2}+\left[J^{\prime \prime}(\omega)\right]^{2}} \\
& G^{\prime \prime}(\omega)=\frac{J^{\prime \prime}(\omega)}{\left[J^{\prime}(\omega)\right]^{2}+\left[J^{\prime \prime}(\omega)\right]^{2}}
\end{aligned}
$$

The calculation of the dynamic moduli via Eq. (11) requires knowledge of the dynamic compliance. The material function measured by the MVM is the time-dependent creep compliance $J(t)$ : therefore, the next step is the conversion between $J(t)$ and $J^{*}(\omega)$. Using Eq. (5) this conversion is trivial when the discrete retardation spectrum $\left\{J_{k}, \tau_{k}, J_{0}, \eta_{0}\right\}$ is known.

It is impoant to note that the conversions following the calculation of the retardation time spectrum (Eqs. (5), (11)) are exact and hence introduce no additional numerical error. Therefore the quality of the whole interconversion pivots on the quality of the calculation of the retardation spectrum from the experimental creep compliance data.

Different paths for the numerical interconversion and between $J(t)$ and $G^{*}(\omega)$ have been suggested in the research literature (Mead, 1994; Tschoegl, 1989; Schwarzl, 1990; Ferry, 1980). We believe that the path via the retardation spectrum described above has the highest precision and the least numerical error. The crucial step in this interconversion process is the determination of the retardation spectrum. In this work a special modification of regularization, the nonlinear regularization, is used. There are three principal reasons for choosing regularization algorithms to solve the ill-posed interconversion problems in our work. First, by comparing different numerical techniques, Brabec et al. (1997) demonstrated that regularization is a robust method capable of calculating physically correct spectra with very high retardation or relaxation time densities. Second, Mead (1994) and Honerkamp and Weese (1989) showed that regularization techniques are able to calculate the correct spectrum even when there is large experimental error in the input data. Investigations on numerically simulated data show that regularization can remove up to $10 \%$ random error from experimental data when recalculating the viscoelastic material function from the spectrum. Third, regularization techniques are 
Table 1 Material characteristics of the commercial polymers examined in this work

\begin{tabular}{llll}
\hline & $\begin{array}{l}\text { MFR } \\
{[\mathrm{g} / 10 \mathrm{~min}]}\end{array}$ & $\begin{array}{l}\rho\left(23^{\circ} \mathrm{C}\right) \\
{\left[\mathrm{g} / \mathrm{cm}^{3}\right]}\end{array}$ & $\begin{array}{l}\log \eta_{0} \\
{[\mathrm{Pas}]}\end{array}$ \\
\hline Daplen DE3964 & 0.80 & 0.939 & $5.76\left(160^{\circ} \mathrm{C}\right)$ \\
Hostyren PSN7000 & $\left(190^{\circ} \mathrm{C}, 5 \mathrm{~kg}\right)$ & 1.05 & $4.88\left(200^{\circ} \mathrm{C}\right)$ \\
Eltex PE91C & 11.4 & 0.944 & $5.84\left(180^{\circ} \mathrm{C}\right)$ \\
\hline
\end{tabular}

able to determine the correct spectrum even in the case of incomplete input data sets, which is a common problem in the determination of viscoelastic properties of polymers with a broad molar mass distribution.

\section{Materials}

Three polymers representative of polydisperse commercial systems were investigated in this study:

- Daplen DE 3964 (HDPE, PCD Polymere GmbH)

- Eltex PE 91C (HDPE, Solvay SA)

- Hostyren PS N 7000 (PS, Hoechst AG)

General material characteristic data are summarized in Table 1. All materials have been supplied by the producers in pellet form and were compression molded for the rheological investigations $(7 \mathrm{~mm}$ rods in the case of the MVM, $2 \mathrm{~cm}$ plaques in the case of the dynamic measurements). The samples were dried prior to measurement.

\section{Experimental}

Three different types of experimental methods were used to generate mastercurves over a wide dynamic range as outlined above. Specifically,

1) Dynamic/mechanical measurements on Rheometrics RDS II (stress-controlled) and Rheometrics CSR (strain-controlled) to determine $G^{\prime}(\omega)$ and $G^{\prime \prime}(\omega)$ in the range of $10^{-2}$ and $10^{3} \mathrm{rad} / \mathrm{s}$.

2) Creep-compliance measurements on Rheometrics RDS II to determine $J(t)$.

3) Constant stress flow startup-measurements around a sphere using a Paar/Physica Magnetoviscosimeter (MVM) to determine $J(t)$.

In the first experiment, a cone-and-plate geometry is used and the relevant standard (ISO 1994) is followed closely. Since there were no significant differences between the moduli data from the two instruments employed, only the RDS II measurements were actually used. In the second experiment, a plate-plate geometry was used for the $J(t)$-measurements on the Rheometrics RDS II.

The creep measurements on the MVM were performed in the following manner: During the sample preparation, a $3 \mathrm{~mm}$ steel ball is centered in the $7 \mathrm{~mm}$ PP-rod. The premolded sample is heated up in a brass cell until a homogeneous temperature-distribution is reached. For the samples investigated, the viscosity is sufficiently high that displacement of the steel ball due to gravity is negligible. When the sample is brought into the magnetic field the steel ball moves under the action of the force $F_{m}$. The displacement of the sphere is related to the shear strain $\gamma(t)$ and is recorded as a function of time. Thus the creep compliance can be derived directly from this curve. Details about the operating principles of the MVM are reported by Gahleitner and Sobczak (1991) and Ringhofer et al. (1996).

\section{Results and discussion}

MVM-creep data have been measured over time ranges typically from 1 to $15000 \mathrm{~s}$. The measurement temperature for the polystyrene sample $\mathrm{N} 7000$ is $200^{\circ} \mathrm{C}$, for the DE3964 $160^{\circ} \mathrm{C}$ and for the PE91C $180^{\circ} \mathrm{C}$. Timetemperature superposition shifts have not been evaluated, as this effect is insufficient for the investigated materials (except the N7000). Since the experimental point density from MVM measurements with 10 points per second is too high for computational investigations, the experimental data set has been culled to point densities of between 20 to 100 points per time decade. The culled MVM creep measurements are overlaid onto $J(t)$ measurements from the DSR rheometer to generate a mastercurve. The mastercurves are shown in Figs. 1, 3 and 4 in a $\log -\log$ format together with the creep curves recalculated from the retardation spectrum.

Typically, the retardation spectrum is calculated by minimization of Eq. (8) with retardation time densities of between 5 to 10 logarithmically equidistant times per decade. The calculations were performed on Pentium PC with typical calculation times of less than $10 \mathrm{~min}$. 


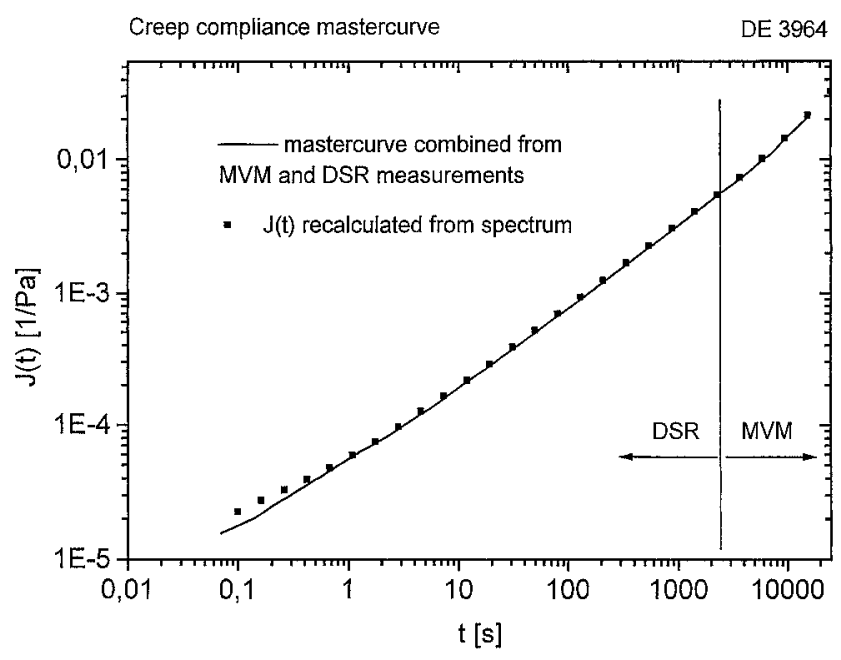

Fig. 1 Creep compliance mastercurve for Daplen DE 3964 at $180^{\circ} \mathrm{C}$ (full squares Rheometrics DSR II and PAAR/Physica MVM, line recalculated from retardation spectrum)

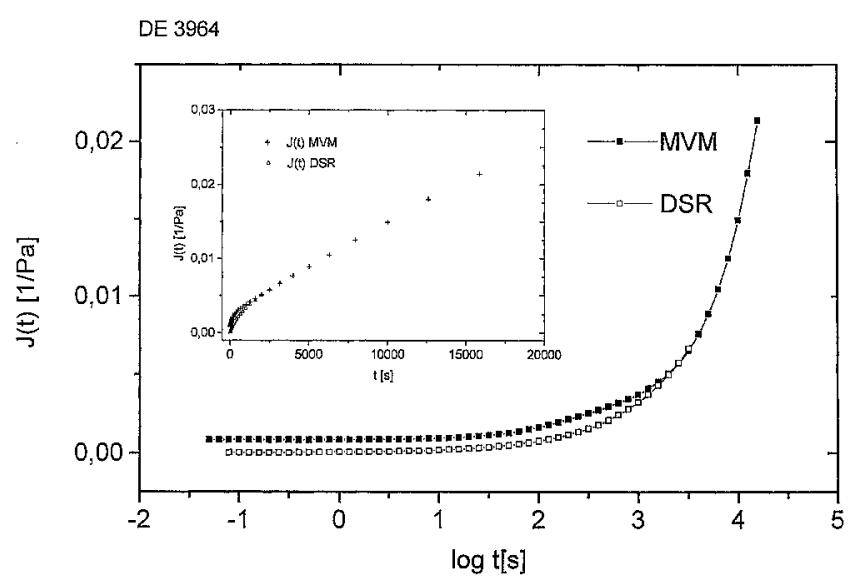

Fig. 2 Comparison of creep measurements for Daplen DE 3964 at $180^{\circ} \mathrm{C}$ (full squares and crosses PAAR/Physica MVM, open symbols Rheometrics DSR II, plate-plare arrangement)

From the calculated parameter set $\left\{J_{k}, \tau_{k}, J_{0}\right.$ and $\left.\eta_{0}\right\}$ the original data are recalculated and compared with the original experimental data. Finally, from Eq. (5) the dynamic creep compliance is calculated.

Figure 1 shows the creep mastercurve of the DE3964 determined by MVM measurements respectively DSR measurements. The arrows indicate the contribution of the single measurement techniques to the mastercurve. The exact correlation time between the different measurements can be seen in Fig. 2. Here the complete creep data as determined by both the MVM and the DSR rheometer are plotted in a double logarithmic representation. For creep times in excess of about $2000 \mathrm{~s}$, the MVM and DSR data are in close agreement. We have selected this creep time as the transition point for the generation of the mastercurve, i.e. at shorter times the creep mastercurve is built by the DSR data, while at

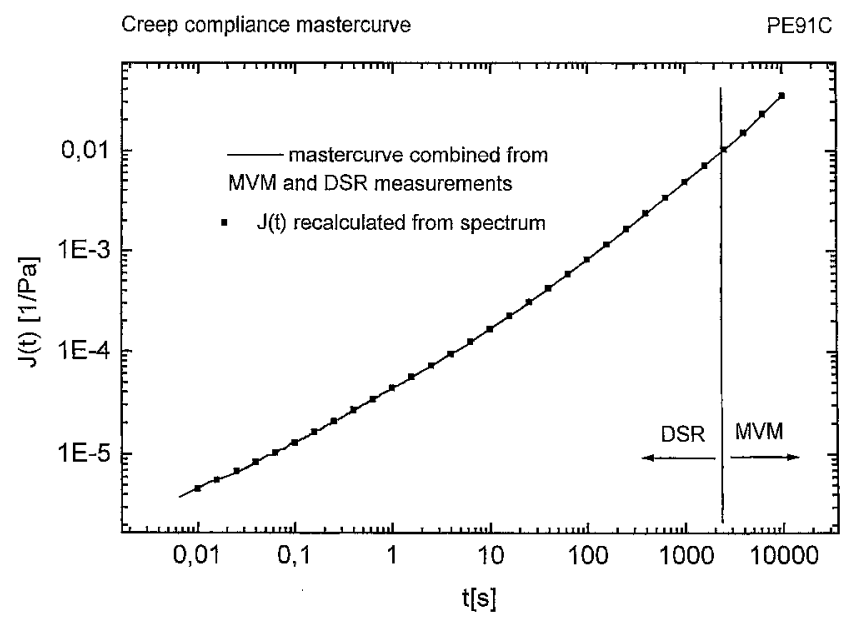

Fig. 3 Creep compliance mastercurve for Eltex PE $91 \mathrm{C}$ at $160^{\circ} \mathrm{C}$ (full squares Rheometrics DSR II and PAAR/Physica MVM, line recalculated from retardation spectrum)

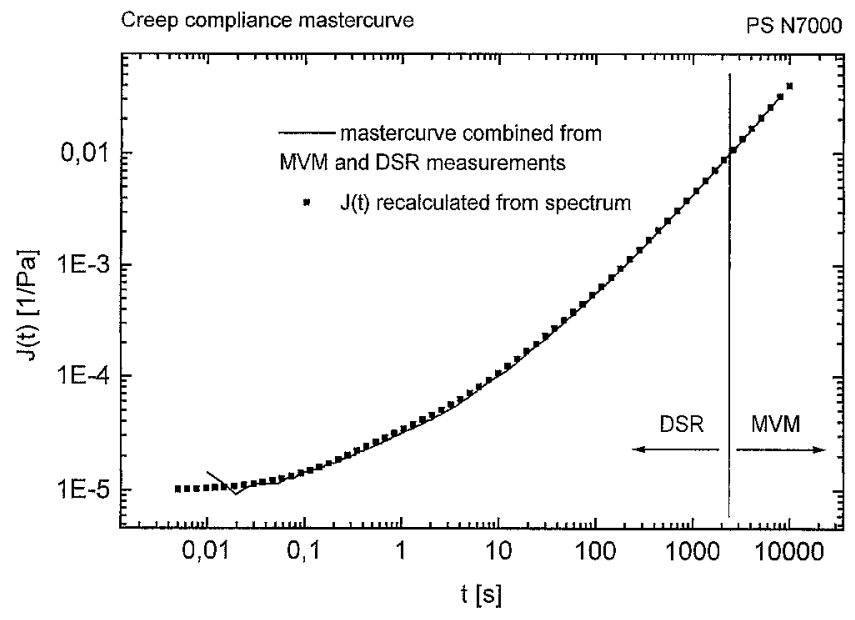

Fig. 4 Creep compliance mastercurve for Hostyren PS N 7000 at $200^{\circ} \mathrm{C}$ (full squares Rheometrics DSR II and PAAR/Physica MVM, line recalculated from retardation spectrum)

times higher than the correlation time only MVM data are used. Using the MVM creep data the window of time scales that could be probed in the creep experiment was expanded by about one decade. This is an especially useful tool for polymers such as polyethylene where time temperature superposition is ineffective in expanding the effective time scale.

Both curves reach the linear flow regime of the creep curve, which is characterized in a double-logarithmic plot by the slope $d \log J(t) / d \log (t)=1$. The onset to the rubbery plateau regime is barely discernible which is typical of systems with large levels of polydispersity. In the very short time region of Fig. 1, one can see small deviations between the experimentally determined creep curve and the recalculated spectrum curve. We attribute these differences to the well known startup problems of creep measurements (Schwarzl, 1990), 


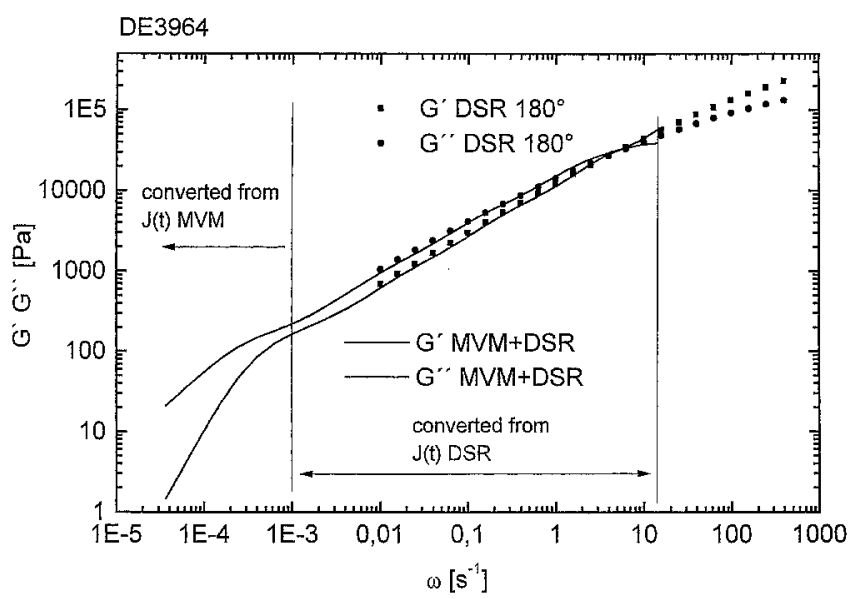

Fig. $5 G^{\prime}, G^{\prime \prime}(\omega)$ for Daplen DE 3964 at $180^{\circ} \mathrm{C}$ (symbols for Rheometrics DSR II, cone-plate arrangement, lines converted from creep compliance mastercurve)

which cannot be described by a generalized Voigt model. Lack of sensitivity and resolution to short time scale phenomena is a well known weakness of controlled stress experiments.

The creep mastercurve of the PE 91C shown in Fig. 3 is very similar to that of the DE 3964 . The correlation creep time between the MVM and the DSR curves is around $2500 \mathrm{~s}$, comparable to that of the DE3964. Both curves reach the linear flow regime, while the rubbery plateau regime is poorly characterized.

For the polystyrene N7000 the rubbery plateau of the creep compliance plotted in Fig. 4 is better characterized than for the polyethylene samples. Again, both creep curves reach the linear flow regime and the correlation between experimentally determined and numerically recalculated mastercurve is excellent. The correlation creep time, marked by arrows in Fig. 4 where MVM and DSR measurements begin to overlay is around $2500 \mathrm{~s}$. At the shortest creep times the compliance is corrupted by the transient startup conditions, leading to small oscillations, seen in Fig. 4, of around $0.1 \mathrm{~s}$. Regularization filters these unphysical oscillations out and the creep compliance recalculated from the retardation spectrum shows the expected smooth shape.

From the retardation spectra of the creep mastercurves the dynamic moduli are calculated as outlined above. The results for the numerical interconverted dynamic moduli are compared to experimental dynamic measurements on the DSR II in Figs. 5-7.

Figure 5 shows the results for the dynamic moduli for the DE3964. The experimentally determined dynamic moduli are plotted as full symbols while the numerically converted data are plotted as continuous curves. It is clear that agreement is excellent in a circular frequency range for more than two decades, indicated by the arrows. It can be seen that the predictions

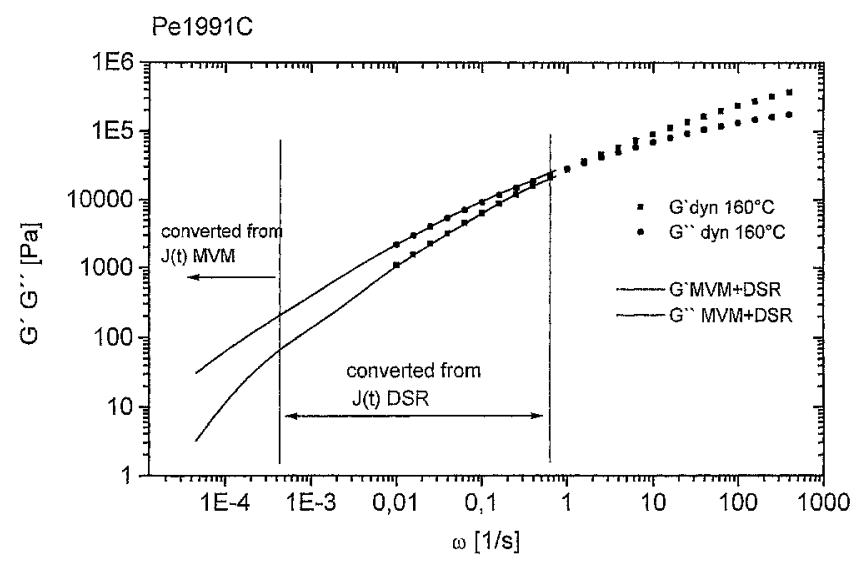

Fig. $6 G^{\prime}, G^{\prime \prime}(\omega)$ for Eltex PE $91 \mathrm{C}$ at $160^{\circ} \mathrm{C}$ (symbols for Rheometrics DSR II, cone-plate arrangement, lines converted from creep compliance mastercurve)

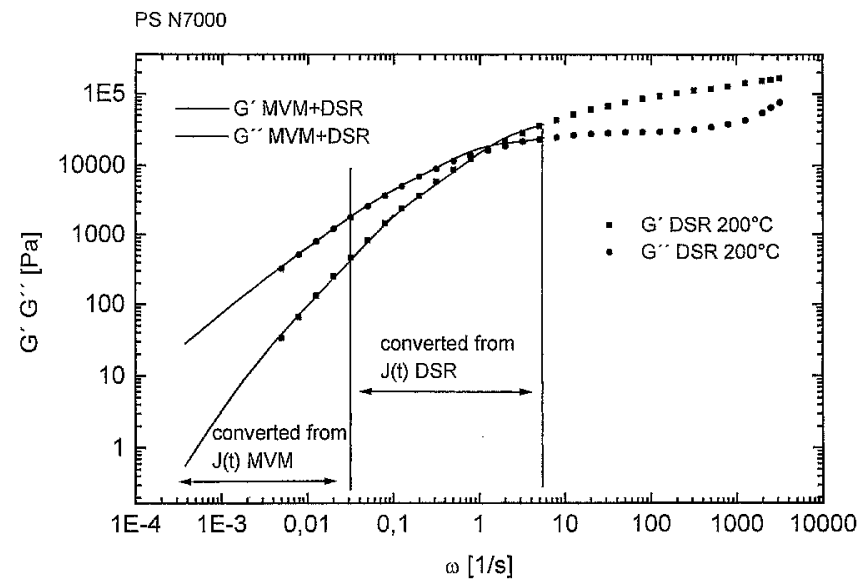

Fig. $7 G^{\prime}, G^{\prime \prime}(\omega)$ for Hostyren PS N 7000 at $200^{\circ} \mathrm{C}$ (symbols for Rheometrics DSR $\Pi$, cone-plate arrangement, lines converted from creep compliance mastercurve)

from the retardation spectrum yield correct moduli up to the crossover point. The small differences for frequencies higher than the crossover point might be explained by the deviation of the recalculated creep mastercurve in the short time range as seen in Fig. 1. The interconversion of creep data expands the dynamic frequency range more than two decades in the low frequency range. The specific contribution of the MVM data to this dynamic mastercurve is about one additional decade in frequency. The predictions of the interconverted MVM data reach fully terminal behavior, characterized by the slopes 1 and 2 for the loss modulus and the storage modulus, respectively, on a doublelogarithmic plot. Thus, the entire terminal zone has been characterized through the longest relaxation time scale (highest molecular weight component).

The results for the PE 91C plotted in Fig. 6 confirm the results found for the DE3964. The agreement be- 
tween measured moduli and moduli calculated via the retardation spectrum interconversion is excellent, even at the shortest creep times (i.e. highest frequencies). An overlay range for more than two decades is found. However, at low frequencies slopes 1 and 2 characterizing the linear flow regime are not reached, indicating that even with the MVM, fully terminal behavior was not achieved for this system.

For the polystyrene N7000, the interconverted and measured moduli agree for three decades in frequency, establishing once more the utility of the numerical interconversion via retardation spectra in expanding the dynamic range of rheological measurements. As with other systems, the MVM measurements contribute to the mastercurve for more than one decade in the low frequency range. The MVM contribution is valuable to more fully describe the terminal zone behavior. Slopes of 1 and 2 of the dynamic moduli indicate that the fully terminal flow regime has been reached.

\section{Conclusions}

We have demonstrated that numerical interconversion of linear viscoelastic material functions is a sound method to effectively expand the experimentally accessible time or frequency range. This method is applicable to arbitrary linear viscoelastic material functions since, in principle, all linear viscoelastic material functions contain equivalent information. The ability to expand the effective dynamic range of rheological measurements has assumed a greater importance in light of the intrinsic theoretical limitations involved in calculating the relaxation or retardation spectrum from dynamic moduli data recently reported by Anderssen and Davies (1997).

Although all linear viscoelastic material functions contain equivalent information, in principle, certain measured material functions are inherently more sensitive and easier to access in different time/frequency regions than others. Creep measurements are most sensitive to long time scale relaxation processes, whereas dynamic moduli measurements are more sensitive to short time scale relaxation processes. Thus interconverting material functions not only increases the dynamic range, it also improves the accuracy of the retardation spectrum calculation.

By combining two different creep measurements with dynamic moduli measurements and interconverting, we have been able to expand the experimentally accessible dynamic range of the dynamic moduli by several decades in the low frequency regime. Specifically, we combined MVM and DSR creep data to generate creep mastercurves. The agreement between creep data from these two different measurement techniques is satisfactory. In the short time range DSR creep data deliver more precise results whereas MVM creep data are more reliable at longer times. Combining the data leads to an expanded range of accessible time scales.

The interconversions of the creep data to dynamic moduli proceeds through the retardation spectrum. To overcome the ill-posed nature of spectra calculations we use a particular form of regularization, specifically nonlinear regularization (Weese, 1992). This robust and reliable numerical method has been successfully used to determine the retardation as well as relaxation spectra. Once a spectrum is obtained, all other linear viscoelastic material functions can be calculated exactly.

For our investigation we chose three polymers with polydisperse molar mass distributions, two HDPEs and one polystyrene, which are representative of typical commercial polymers. Comparison of interconverted and directly measured dynamic moduli shows excellent agreement for all investigated materials in the frequency range where the curves overlap, confirming both the high quality of the creep measurements and the subsequent numerical calculation of the retardation spectra. Due to their broad molar mass distributions, the terminal flow regime of these materials is rather poorly characterized by the experimentally determined dynamic moduli. For all three materials, the experimental dynamic moduli do not reach the terminal zone which is a consequence of intrinsic mechanical limitations of the experiment, specifically the limited head space of the torque transducer. Expansion of the dynamic range with the help of converted creep data allows one to gain access to more detailed information in the terminal regime and, more importantly, an effective characterization of the high molecular weight tail of the MWD. For two of our polymers the fully terminal slopes of 1 and 2 for the converted loss and storage modulus in the doublelogarithmic plot were reached.

Our data lead us to draw three basic conclusions. First, MVM creep measurements are well suited for the characterization of the long time scale $(t>10000 \mathrm{~s})$ relaxation processes in the linear flow regime. Second, retardation spectra are a viable conduit for the interconversion of linear viscoelastic material functions. Third, the combination of creep compliance and dynamic moduli is a powerful method to expand the experimentally accessible dynamic range of time/frequency and more fully characterize the linear viscoelastic relaxation and retardation spectra of polydisperse commercial polymers from the terminal to the glass regime.

Acknowledgements M. Gahleitner from the PCD Linz has generously supplied us with experimental measurements on a DSR II rheometer. 


\section{References}

Anderssen RS, Davies AR (1997) Sample localization in determining the relaxation spectrum. JNNFM (accepted for publication)

Baumgaertel M, Winter HH (1989) Determination of discrete relaxation and retardation spectrá from dynamic mechanical data. Rheol Acta 28:511-519

Brabec CJ, Rögl H, Schausberger A (1997) submitted to Rheol Acta

Brabec CJ, Schausberger A (1995) An improved algorithm for calculating relaxation time spectra from material functions of polymers with monodisperse and bimodal molar mass distributions. Rheol Acta 34:397-405

des Cloizeaux J (1992) Relaxation of entangled and partially entangled polymers in melts: time dependent region. Macromolecules 25:835-841

des Cloizeaux J (1990) Relaxation and viscosity anomaly of melts made of long entangled polymes. Time-dependent region. Macromolecules 23:4678-4687

Doi M, Edwards SF (1986) The Theory of Polymer Dynamics. Clarendon Press, Oxford

Emri I, Tschoegl NW (1993) Generating line spectra from experimental responses. I. Relaxation modulus and creep compliance. Rheol Acta 32:311-321

Emri I, Tschoegl NW (1994) Generating line spectra from experimental responses. IV. Application to experimental data. Rheol Acta 33:60-70

Ferry JD (1980) Viscoelastic Properties of Polymers. John Wiley \& Sons

Franck AJP (1985) A rheometer for characterizing polymer melts and suspensions in shear creep and recovery experiments. J Rheol 29:833-850
Friedrich C, Hoffman B (1983) Nichtkorrekte Aufgaben in der Rheometrie. Rheol Acta $22: 425-434$

Gahleitner M, Sobczak R (1991) The magnetoviscometer: from an idea to a rheological instrument. Rheology 91:236-240

Honerkamp J, Weese J (1989) Determination of the relaxation spectrum by regularization method. Macromolecules 22:43724377

Honerkamp J, Weese J (1993) A nonlinear regularization method for the calculation of relaxation time spectra. Rheol Acta 32:65

Honerkamp J, Weese J (1990) Tikhonov's regularization method for ill posed problems. Continuum Mech Thermo 2:17-30

Honerkamp J, Weese J, Elster JC (1991) Using regularization methods for the determination of relaxation and retardation spectra of polymeric liquids. Rheol Acta 30:161-174

Janeschitz-Kriegl H (1983) Polymer Melt Rheology and Flow Birefringence. Springer, Berlin

Kaschta J (1992) On the calculation of discrete retardation and relaxation spectra. In: Moldenaers P, Keunings $R$ (eds) Theoretical and Applied Rheology, Vol 2, p 155. Elsevier, Amsterdam

Kaschta J, Schwarzl FR (1994) Calculation of discrete retardation spectra from creep data - I. Method. Rheol Acta 33:517-529

Kaschta J, Schwarzl FR (1994) Calculation of discrete retardation spectra from creep data - II. Analysis of measured creep curves. Rheol Acta 33:530-541

Mead DW (1994) Numerical interconversion of linear viscoelastic material functions. J Rheol 38 (6):1769-1795
Mead DW (1994) Determination of molecular weight distributions from linear viscoelastic material functions. J Rheol 38 (6): 1797-1827

Morozov VA (1984) Methods for solving incorrectly posed problems. Springer, Berlin New York

Plazek DJ (1992) What's wrong with the moduli Charlie Brown? or Get the $\mathrm{H}$ out and go to L. J Rheol 36:1671-1680

Plazek DJ, Chay IC (1991) The evolution of the viscoelastic retardation spectrum during the development of an epoxy resin network. J Poly Sci: Phys ed 29:17-29

Provencher SW (1982) CONTIN: A general purpose constrained regularization program for inverting noisy linear algebraic and integral equations. Comput Phys Commun 27:229-242

Ringhofer R, Gahleitner M, Sobczak R (1996) Low frequency/shear rate measurements on polymer melts with a novel rheometer. Rheol Acta 35:303-307

Schwarzl FR, Staverman A (1952) Higher approximations of relaxation spectra. Physica 18:791-799

Schwarzl FR (1990) Polymermechanik. Springer, Berlin

Thayer AM (1995) Metallocene catalysts initiate new era in polymer synthesis. C\&E News 73:15

Tschoegl NW (1989) The phenomenological theory of linear viscoelastic behavior. Springer, Berlin

Tsenoglou C (1991) Molecular weight polydispersity effects on the viscoelasticity of entangled linear polymers. Macromolecules 24:1762-1767

Weese J (1992) Datenanalyse in der Rheologie mit Hilfe von Regularisierungsverfahren. $\mathrm{PhD}$ Thesis, University of Freiburg 